\title{
Juventud, enseñanza secundaria y trayectorias de vida
}

\author{
Oscar Dávila León*
}

Felipe Ghiardo Soto**

\section{Resumen}

El presente texto recorre algunos de los cambios que está produciendo la instalación de la escolarización como mecanismo legítimo de posicionamiento social entre los jóvenes estudiantes del sistema de educación municipal secundario en Chile. La tesis que se plantea es que este proceso está cambiando las estrategias de reproducción en estos grupos sociales, que se expresa en la configuración de proyectos de vida tanto en el plano de las trayectorias escolares como en el de las formas de transitar hacia la adultez.

Palabras clave: Escolarización; Transición; Trayectorias; Condiciones juveniles.

\section{Youth, high school, and life paths}

\section{Abstract}

The present text covers some of the changes that the installation of schooling as legitimate mechanism of social positioning among young students from the municipal secondary education system in Chile is producing. The thesis is that this process is changing the strategies of reproduction in these social groups, which expresses itself both in the configuration of life projects in the plane of educational paths and in one way to move into adulthood.

Keywords: Education; Transition; Paths; Juvenile conditions.

\footnotetext{
* Professor trabajador social y doctorando en el estudio de las sociedades latino: Centro de Estudios Sociales CIDPA - Valparaíso - Chile.

${ }^{* *}$ Professor licenciado en sociología, diplomado en juventud y políticas sociales, mención sociología de la modernización. Investigador del Centro de Estudios Sociales CIDPA - Valparaíso - Chile.
} 


\begin{abstract}
La escuela no es simplemente un lugar donde se aprenden cosas, saberes, técnicas, es también una institución que otorga títulos - es decir, derechos - y confiere al mismo tiempo aspiraciones. El antiguo sistema escolar producía menos confusión que el sistema actual con sus ramificaciones complicadas, que provocan que las personas tengan aspiraciones mal ajustadas a sus oportunidades reales.
\end{abstract}

Pierre Bourdieu, La 'juventud' sólo es una palabra, 2000.

Dentro de quienes se han ocupado de estudiar "lo juvenil" en Chile durante las últimas décadas, y en especial a lo concerniente a estudiantes secundarios, se generó una suerte de imagen discursiva que intentaba vincular a estas juventudes actuales, a los hijos e hijas de la transición política chilena, los nacidos alrededor del año 1990, con la reinauguración de la democracia y el término de la dictadura militar. Se decía que estábamos en presencia de la primera generación contemporánea de jóvenes que no tenía ninguna "misión histórica que cumplir", que no traía consigo "ninguna mochila pesada que cargar". Todo esto expresado desde la clásica visión sociopolítica con que nos hemos acostumbrado a leer los procesos sociales.

Como hipótesis podemos adelantar que estaríamos en presencia de un nuevo sujeto joven estudiante secundario, quien ha interiorizado - en lo discursivo y fáctico - una resignificación y elevación de expectativas y aspiraciones (no sólo en materia de metas educacionales, sino que como configuradoras de proyectos de vida), que desde su percepción, no tendrían posibilidades de ser cumplidas por el sistema social y educativo, en cuanto a garantizar las oportunidades sociales. En dos palabras: el desface entre altas expectativas y bajas posibilidades. A su vez, también interesa el trazar algunas conexiones entre los jóvenes estudiantes y los otros actores del sistema educativo, como la escuela y los docentes, e interrogarnos sobre los posibles roles y desafíos que éstos pueden encarar en perspectiva de favorecer y colaborar con la concreción de los proyectos educativos y vitales de los jóvenes. Para eso nos remitiremos fundamentalmente al estudio realizado por CIDPA con jóvenes del sistema municipalizado (DÁVILA, GHIARDO Y MEDRANO, 2006).

\title{
Para entender los nuevos sujetos y sus procesos
}

La teoría pedagógica asume que la labor educativa implica siempre a un sujeto: el sujeto de educación. Los distintos enfoques pedagógicos difieren en su objetivo y forma de trabajo dependiendo de cómo definan a ese sujeto, de qué cualidades le atribuyan. La tendencia más simple ha sido definirlo en base a sus características comunes, y la más inmediata y evidente para la pedagogía tradicional ha sido la etaria. Se trataría fundamentalmente de niños y niñas en el caso de la educación básica, y de adolescentes/jóvenes en la educación media. En estas dos categorías se sostiene la construcción más "clásica" de los 
planes y políticas educativas: distribución de contenidos por niveles, cursos distribuidos por edad.

Producto del desarrollo de las ciencias sociales y también de la propia reflexión y trabajo pedagógico (los profesores se dan cuenta de la diferencia entre niños y niñas, o entre niños "ricos" y "pobres"), se ha hecho evidente que esta sola categoría resulta insuficiente para definir a los sujetos de educación, fundamentalmente porque reduce la vida a una sola dimensión (la edad) y deja en segundo plano un conjunto de condiciones (sociales, económicas, demográficas, culturales, de género) que son tanto o incluso más importantes. La idea de adaptar los currículos a la realidad o a las características de los sujetos, que aparece como uno de los principios centrales para la reforma que se inició el año 1996 en Chile, intenta de algún modo hacerse cargo de esta falencia.

Frente a estas insuficiencias lo que aparece como más pertinente es adoptar lo que se puede definir como una perspectiva generacional, un enfoque que mire desde distintos ángulos, que cubra las características más evidentes, pero que las ponga a contraluz y las ubique en un contexto (sociocultural) y las observe en movimiento (histórico), para de ese modo ampliar el abanico de posibilidades de análisis de lo que ocurre hoy con el sujeto de educación de los establecimientos públicos municipalizados.

Para ello podemos seguir varias pistas. Entre ellas, la primera y que a esta altura ya es de dominio público, tiene que ver con uno de los efectos perversos de la Ley Orgánica Constitucional de Enseñanza (LOCE) y la masificación de la enseñanza formal: la progresiva concentración en el sistema municipalizado público de jóvenes que provienen de los sectores con menos recursos, que son al mismo tiempo los grupos o clases cuyas generaciones adultas históricamente han tenido pasos menos prolongados por la escuela. Solamente un par de antecedentes. Según la Encuesta Nacional de Caracterización Socioeconómica (CASEN), más del $72 \%$ de la población que asistía a la educación media en establecimientos municipales públicos pertenecía a los primeros cinco deciles de ingresos, que corresponden precisamente a los de menores ingresos (MIDEPLAN, 2004; 2010).

Otro dato que se corresponde con el anterior: la escolaridad de los padres de estos jóvenes tiende a ser baja, en su mayoría con menos de doce años de escolaridad, y una porción importante que no alcanzó a completar la educación básica. Son muy pocos los jóvenes que son hijos o hijas de padres y madres profesionales (bordean el $12 \%$ ), y además tienden a concentrarse principalmente en los establecimientos de mayor "tradición" de las distintas ciudades, que suelen corresponder más a liceos que imparten la modalidad Científico Humanista $(\mathrm{CH})$ que la Técnica Profesional (TP). 
Veamos ahora otras dimensiones que también son ilustrativas. Si analizamos los grados de integración a las Tecnologías de la Información y Comunicación (TIC), que viene siendo uno de los mecanismos de integración cultural más potentes, sobre todo para la población joven, encontramos que la porción de estudiantes que maneja el lenguaje computacional más básico sigue siendo reducida ( $47 \%$ según los datos que manejamos), la que tiene computador en su hogar es todavía bastante pequeña (cerca de un 30\%), y menor aún la que tiene acceso a la red internet (12\%). La carencia de equipos en el hogar es claramente un factor de desventaja, pues quienes sí lo tienen muestran un nivel de "alfabetización digital" notablemente superior al de quienes no están en la misma condición. A estos últimos las posibilidades que les quedan son los computadores de los propios liceos y de otros lugares extraescolares (telecentros, cibercafés), pero en el primero no siempre se puede o no siempre dejan, y en los segundos hay que pagar.

En la misma línea, pero esta vez en términos de "consumos culturales", de acuerdo a las variables que exploramos, podemos sostener que entre los jóvenes que asisten al sistema municipalizado son en realidad bastante pocos los que se despegan de una tendencia generalizada a mantenerse alejados de las prácticas de consumo cultural "culto" (leer las "grandes obras" de la literatura, leer la historia, ir al cine o a otros espectáculos artísticos, etc.), aquellas prácticas que configuran la imagen de la "persona culta" y que se esmera en serlo. Por el contrario, más parece dominar una distancia hacia estas prácticas, incluso con un grado de ironía hacia quienes sí las asumen, que suelen ser los "mejores alumnos", los "mateos", en una actitud que algunos autores (MCLAREN, 1998; APPLE, 1987) han interpretado como parte de los mecanismos de resistencia que los estudiantes de sectores desfavorecidos desarrollan en el cotidiano frente a la imposición cultural que encierran los contenidos que transmite la institución escolar. La gran mayoría se apega a los consumos estandarizados que ofrecen los medios de comunicación masivos, y por lo general, son pocos los que han tenido en el último tiempo posibilidades de vivir experiencias que ayudan a construir una imagen y visión "más amplia" del mundo, como viajar más allá de los límites de la región, porque en general son prácticas que requieren tiempo y recursos.

Todas estas variables que hemos revisado son variables "estructurales" que de algún modo ayudan a graficar la posición de los jóvenes y la de sus familias en la estructura social. En este sentido representan un buen punto para comprender los marcos en que se produce la existencia y la subjetividad de estos jóvenes, para a partir de ahí, tratar de entender las diversas lógicas que buscan los jóvenes para darle curso a su vida. Y es que los jóvenes están en medio de un período complejo en que todo se conjuga para que su relación con el mundo adquiera otros matices y se dé en otros términos. En el período que corresponde a la educación secundaria les llegan transformaciones por todos lados, por procesos de distinto orden: cambia su constitución biológica y su condición como sujetos sociales, se les otorga grados mayores de autonomía, 
van definiendo sus propios intereses, su propia identidad, se identifican con grupos, con actividades, con discursos, participan de "espacios de jóvenes" (fiestas, carretes, tocatas), establecen relaciones amorosas, algunos transgreden normas culturales. Y por si fuera poco, socialmente, por la acción del sistema escolar mismo, que es uno de los espacios donde más tiempo pasan, se les obliga a tomar decisiones difíciles que les pone de cara a su futuro personal.

Una de ellas es decidirse por una modalidad de estudios. Hay que optar por un camino, el Científico Humanista o el Técnico Profesional. En principio y también en la práctica, una y otra alternativa conducen a destinos diferentes: por un lado la esperanza de que los estudios secundarios sean un puente de paso hacia la educación superior; por el otro, la posibilidad de obtener un oficio certificado al terminar la educación media que permita ingresar al mundo del trabajo o adelantar camino en la definición de un área de especialización. La decisión es compleja, hay que calibrar muchos factores. Lo peculiar es que el sistema escolar chileno ha venido reservando este dilema casi exclusivamente a quienes estudian en el sistema municipalizado público. Prácticamente no existen establecimientos privados que impartan la modalidad técnica y son muy pocos los particulares subvencionados por el Estado. La gran mayoría de las carreras técnicas se imparten en establecimientos municipalizados públicos, y su avance ha sido tal que si hasta un par de décadas atrás la modalidad Científico Humanista era ampliamente mayoritaria, hoy en día está prácticamente a la par con la Técnico Profesional. Sobre este punto convergen varios factores, principalmente extraescolares: la necesidad de la industria y el comercio de contar con un contingente capacitado, el impulso discursivo a la modalidad TP por parte de los representantes políticos de la educación, el aumento de la "oferta" TP con la inauguración de nuevos establecimientos y la conversión de establecimientos $\mathrm{CH}$ en TP. Pero también influyen factores que están a nivel subjetivo, que tienen que ver con el modo en que el sujeto procesa estos acontecimientos y delinea su proyecto de vida.

Con esto llegamos a la segunda decisión, quizás más compleja, pero ligada a la anterior: la opción por un camino para cuando concluya la educación media. Aquí adquiere todo su peso el discurso social que ubica a los estudios como principal promesa de movilidad social, como la alternativa (ideal) para "ser alguien en la vida". Es complejo explicarlo, sobre todo si tenemos en cuenta que las tendencias macrosocial demuestran la desigualdad de destinos dependiendo del tipo de establecimiento en que se estudia o la clase a la que se pertenece, pero la potencia y legitimidad del discurso escolar parece no cuestionarse. $Y$ es que en general la gran mayoría de los jóvenes pone a los estudios como un componente esencial a la hora de definir su proyecto de vida.

No deja de ser importante el porcentaje que piensa dejar como tope de escolaridad la educación secundaria completa (22\%), que en líneas generales tiende a ser más frecuente entre los hombres que las mujeres, entre quienes asisten a la modalidad TP que a la $\mathrm{CH}$, y entre quienes pertenecen a familias 
con menores niveles de escolaridad; la mayoría se proyecta hacia el campo de los estudios superiores. Entre éstos hay diferencias en el modo en que proyectan ese paso a la educación superior: si bien la proporción más alta quisiera un paso inmediato y concentrado solamente en los estudios superiores (36\%), no es menor la porción que se anticipa forzado a combinar trabajos y estudios $(23 \%)$, o a postergarlos para antes trabajar un tiempo (10\%), seguramente pensando en una forma de juntar recursos y experiencia.

Si vemos las distintas alternativas que contempla el sistema de educación superior, la que concentra la mayor parte de las aspiraciones es la Universidad. En realidad, es difícil que no lo fuera si tenemos en cuenta que la figura de esta institución aparece como un símbolo que representa más que ninguna otra la posibilidad de vivir un "ascenso" social y cultural significativo. Al menos idealmente, muchos quisieran ser profesionales de algún campo bien posicionado, algo que les asegure el futuro y les permita surgir o superar las condiciones en que ha transcurrido su experiencia personal y la historia de sus familias. En un segundo lugar y varios puntos más abajo, aparecen los Institutos Profesionales y, más atrás, los Centros de Formación Técnica, que son la alternativa en principio menos considerada, aunque en la práctica constituyan uno de los destinos más frecuentes para la población de los quintiles de más bajos ingresos (MIDEPLAN, 2004).

Si nos ponemos a ver las diferencias en el nivel de las metas escolares que se proyectan, se observa que pasan fundamentalmente por la modalidad de estudios que se encuentran cursando y el género. En términos generales, quienes siguen la modalidad TP tienden a plantearse metas más cortas, con una proporción comparativamente bastante mayor de casos que aspira dejar como tope la educación media y su título de carrera como herramienta de inserción laboral, y con una menor proporción de casos que aspira llegar a la universidad. En un sentido, se percibe cierto conformismo con la formación para el trabajo, que según lo han interpretado algunos autores (BOURDIEU, 1988; WILLIS, 1988) no sería sino el rastro de la ideología de la clase trabajadora que se actualiza, pero que a la larga no hace más que ayudar a reproducir la estructura de clases pre-existente.

Lo que ocurre con el género es destacable, principalmente por las marcadas diferencias que se producen entre las opciones de futuro que se vienen planteando las mujeres y los hombres. Es un dato notable que tanto las proporciones que aspiran a seguir estudios superiores como las que aspiran completar estudios universitarios sean considerablemente mayores entre las mujeres que entre los hombres. Mientras de los hombres el $26 \%$ aspira ingresar a la universidad, entre las mujeres ese anhelo representa al $55 \%$; es decir, cerca de un $20 \%$ más que entre los hombres. Lo interesante es que en este caso se trata de mujeres jóvenes que pertenecen a los estratos de menos recursos, lo que desde ya encierra un cambio en la "mentalidad femenina" de la mayor importancia. $Y$ es que para las mujeres la alternativa educacional ha demostrado ser la prin- 
cipal herramienta para integrarse de manera efectiva al mundo del trabajo. Por eso en el discurso de las jóvenes estudiantes aparece fuerte la intención de no ser lo que fueron o no vivir lo que vivieron sus madres, y asumir una nueva "imagen ideal" de mujer que tiende a despegarse de la mujer dedicada a las labores del hogar o trabajos mal remunerados para acercarse más a la de mujer independiente, profesional y que participa del mundo laboral, identidades femeninas históricamente más ligadas a la situación de las mujeres en las clases medias y altas que a las de sectores de menores recursos.

Lo interesante de todos estos juegos de proyección respecto a los estudios, estos espacios para "soñar despiertos", es que son construcciones que se ligan directamente a otras "dimensiones de la vida" que en su conjunto permiten distinguir diferentes maneras de ordenar los cursos que se anticipan para sus proyectos de vida. Se puede decir que los estudios actúan como visagra que articula la construcción de esos proyectos. Así por ejemplo, mientras más años se piensa estudiar, más se pretende postergar el paso hacia la autonomía respecto del hogar, más tarde se espera ingresar al mundo del trabajo, menos se piensa en construir una familia o en mayor medida se pretende dejarlo para más tarde. La forma en que se conjuguen estos elementos (estudios/trabajo/ independencia/construc-ción/no construcción de familia) son los que definirán, en el fondo, el modo en que "se hizo la vida", en que el sujeto se construyó a sí mismo.

Pero más allá de esas diferencias en los caminos que se anticipan, que no dejan de ser sólo caminos posibles, lo que es común a todos estos jóvenes es su anhelo de integrarse, de formar parte del juego, pero no en posiciones subalternas que signifiquen carencias e inestabilidad. Si en su discurso los estudios son importantes es porque se mezclan elementos vocacionales ("estudiar lo que me gusta"), elementos pragmáticos ("estudiar algo que me permita surgir"), y elementos que le dan un sentido más abstracto de desarrollo espiritual o moral ("estudiar para ser mejores personas"). Todas estas dimensiones son importantes, pero la tensión que experimentan es porque sienten que no todas se podrán cumplir... o quizá ninguna.

No es casual que si por un lado, e independiente del camino que se quiera tomar, se percibe un ánimo general de optimismo de cara al futuro (INJUV, $2004 ; 2007 ; 2010)$, que en el fondo resume una sensación generalizada de que "les va a ir bien", ellos y ellas saben que nada será fácil, que el mundo no los favorece. De hecho el discurso de los jóvenes del sistema municipalizado público está marcado por el sentimiento de desventaja respecto a los "otros" jóvenes, los que están en los otros tipos de establecimientos. Por eso no resulta extraño que la mayor parte crea que en las actuales condiciones que presenta la sociedad chilena, las posibilidades de concretar sus aspiraciones y hacer realidad sus sueños, son en verdad pocas o simplemente ninguna. Lo complejo es que si por un lado la sensación o a estas alturas, la certeza que sus posibilidades son más bien escasas, hay una tendencia a pensar que son menores mientras 
mayores son las aspiraciones en términos de escolaridad. Así por ejemplo, entre quienes solamente quieren terminar sus estudios secundarios y nada más, hay una proporción menor de jóvenes que piensa tener pocas posibilidades de concretar sus proyectos; mientras entre quienes aspiran llegar a la universidad, es mayor la cantidad de jóvenes que piensa que sus posibilidades son pocas.

Es en estas contradicciones por donde se escurren las desigualdades de todo tipo que impregnan el ambiente en Chile, el modo en que se expresa la fragmentación clasista que se impuso al sistema escolar hechos carne en la persona de estos jóvenes; por eso que ese optimismo se puede leer como un "a pesar de todo, prefiero pensar que me va a ir bien", que más responde a la idea de no cerrarse a la posibilidad de pensar y de construir un futuro, o de no rendirse antes de empezar, que a un optimismo fundado en condiciones, si se quiere, realistas. De ahí que en este desfase entre las aspiraciones y las posibilidades, que se traduce en la distancia entre lo que se quiere o sueña con la realidad y las posibilidades que ofrece, se encuentre a nuestro juicio uno de los nudos críticos por los que atraviesa el sistema educacional secundario en Chile.

\section{Un nuevo sujeto en un nuevo escenario}

Al volver la mirado sobre los jóvenes, podemos establecer que estamos en presencia de adolescentes y jóvenes estudiantes distintos, con características diferentes, tanto en su ser joven como en las formas que expresan y estarían viviendo, percibiendo y significando las diferentes maneras en que asumen sus condiciones juveniles; lo que ha impactado con fuerza en la asunción del rol social u oficio de estudiante. Detrás de aquello hay una permanente intencionalidad en traer las condiciones y culturas juveniles (en plural) al espacio de la cultura escolar (en singular), lo que plantea primariamente la disputa por la pluralidad en contra de la singularidad.

Esta generación de adolescentes y jóvenes estudiantes, en propiedad son "hijos de la transición política a la democracia", muchos nacidos y todos educados en ese contexto/espacio social y político. Sólo han vivido una sociedad democrática; a diferencia de sus padres como "hijos de la dictadura", con todas las valoraciones y significaciones que pueden hallarse a la base de esas experiencias vitales, sobre todo en las referidas a las formas y modos de vivir el período de adolescencia y juventud en uno y otro contexto sociopolítico: y eso no es un antecedente menor.

Estos jóvenes estudiantes son herederos - en sus éxitos y fracasos de la historia reciente, particularmente en el acelerado proceso de modernización (quizá en su tercera fase modernizadora contemporánea) experimentado en la sociedad chilena, lo que ha permitido en buena medida, el poder desplegar esas situaciones y condiciones juveniles a las cuales intentan adscribirse. 
Esta es la generación joven más escolarizada de la historia, que ya exhibe niveles de escolaridad superiores a las de sus padres y madres, los cuales guiaron su vida bajo la promesa hacia sus hijos de dejarles educación y "que sean más que ellos". Esa promesa ya se cumplió, en la gran mayoría, y en los menos, está ad portas de cumplirse. Es la primera generación que está completamente incorporada en la educación primaria, llegando aceleradamente al ciento por ciento en la enseñanza secundaria (94.6\%), y también incorporándose a trancos largos a la educación superior (39.6\% en jóvenes entre 18 y 24 años), pero con muy desiguales accesos de acuerdo a sus condiciones económicas familiares (MIDEPLAN, 2010).

Es una generación que está contenta con la vida que lleva y manifiesta un tremendo optimismo en su futuro y en su vida futura, que adopta un sentimiento de pragmatismo, ven de manera práctica y vivencial si sus expectativas optimistas tienen correlato con sus experiencias de vida. El problema es que ya sospechan del desajuste entre esas altas aspiraciones y expectativas, las que ven difícil de concretar, reconocen que tienen pocas posibilidades para cumplirlas, por los desiguales accesos a las oportunidades sociales, de acuerdo a los orígenes sociales familiares, los bajos capitales heredados y la escasa movilidad social que estaría ofreciendo la sociedad chilena, resultando complejo el compensar o revertir las desigualdades de origen.

Por no haber recibido capitales suficientes, es que se constituyen en desheredados, sujetos que deben extremar esfuerzos para acumular capitales, de preferencia por la vía de una mayor escolarización, lo que podría acercarlos al cumplimiento de sus aspiraciones y expectativas de vida. Ésa es una de las tensiones fundamentales que enfrentan estos jóvenes estudiantes del sistema público de educación: ¿cómo congeniar esas altas expectativas y aspiraciones con sus reconocidas pocas posibilidades? Interrogante que seguirá abierta en busca de respuesta satisfactoria, y que sin duda seguirá interpelando a los distintos actores involucrados en el proceso educativo.

\section{Conclusiones}

La sociedad chilena ha experimentado un acelerado proceso de modernización durante las últimas tres décadas, lo que ha permitido avanzar en la mejoría de ciertas condiciones de vida de la población, siendo éstas tanto materiales como inmateriales, y que cuyos beneficiados en buena medida corresponde a los segmentos juveniles.

Uno de estos procesos modernizadores y también democratizador, sin lugar a dudas ha estado constituido por la dimensión educativa de su población, permitiendo un importante incremento en los accesos a los diferentes niveles del sistema educativo, incluso de sectores sociales que históricamente habían permanecido excluidos o expulsados del acceso, permanencia y egreso de mayores niveles de escolarización. No obstante, con estos procesos no ha 
sido posible revertir en dimensiones significativas la falta de equidad y desigualdades educativas entre los distintos grupos socioeconómicos.

De manera conjunta con esa expansión y elevamiento del capital escolar de la juventud chilena, de la mano a aquélla, ha ido un relevamiento en ciertas condiciones de la subjetividad juvenil en perspectiva de sus trayectorias de vida, donde cada vez tiende a concebirse - desde la dimensión objetiva y subjetiva del sujeto - que la variable que más discrimina y que oficia como mejor predictor del proyecto de vida, está íntimamente asociado a la variable educativa y los grados de escolarización y credenciales educativas obtenidas.

Sobre esta base, comienza a configurarse una suerte de revolución o expansión de las expectativas y aspiraciones juveniles a intentar cumplirse por la vía de la escolarización, particularmente entre los segmentos juveniles de las clases sociales más desfavorecidas. Ese dilema y tensión de la sociedad chilena, expresada en el congeniar ese elevamiento de expectativas con el despliegue de respuestas basadas en posibilidades reales de igualación de oportunidades sociales para los diferentes colectivos societales y juveniles, se constituye en un campo privilegiado de actuación dirigido al mundo juvenil chileno.

\section{Referências}

APPLE, MICHAEL. Educación y poder. Barcelona: Paidós, 1987.

BOURDIEU, PIERRE. Cuestiones de sociología. Madrid: Istmo, 2000. ma, 1997.

Razones prácticas: sobre la teoría de la acción. Barcelona: Anagra1988.

. La distinción: criterio y bases sociales del gusto. Madrid: Taurus,

BOURDIEU,; JEAN-CLAUDE PASSERON. Los herederos: los estudiantes y la cultura. Buenos Aires: Siglo XXI, 2003.

BOURDIEU, P.; JEAN-CLAUDE PASSERON. La reproducción. Elementos para una teoría del sistema de enseñanza. México: Fontamara, 1996.

CASASSUS, J. La escuela y la (des)igualdad. Santiago: LOM, 2003.

DÁVILA, O; FELIPE, G.; MEDRANO, C. Los desheredados: condiciones de vida y nuevas condiciones juveniles. Valparaíso: Ediciones CIDPA (Segunda Edición Aumentada). 2006.

DUBET, F.; MARTUCCELLI, D. En la escuela: sociología de la experiencia escolar. Barcelona: Editorial Losada, 1998.

INJUV. Sexta encuesta nacional de juventud 2009. Santiago: INJUV, 2010.

. Quinta encuesta nacional de juventud 2006. Santiago: INJUV, 2007. 
INJUV. Cuarta encuesta nacional de juventud 2003: la integración social de los jóvenes en Chile 1994-2003. Santiago: INJUV, 2004.

LABARCA, A. Historia de la enseñanza en Chile. Santiago: Publicaciones de la Universidad de Chile, 1939.

LÓPEZ, N. Equidad educativa y desigualdad social. Desafíos a la educación en el nuevo escenario latinoamericano. Buenos Aires: IIPE-Unesco, 2005.

MARTÍN CRIADO, E. Producir la juventud. crítica de la sociología de la juventud. Madrid: Istmo, 1998.

MCLAREN, P. La vida en las escuelas. México: Siglo XXI, 1998.

MIDEPLAN.Encuesta de caracterización socioeconómica nacional. CASEN 2009. Santiago: MIDEPLAN, 2010.

. Encuesta de caracterización socioeconómica nacional. CASEN 2003. Santiago: MIDEPLAN. 2004.

. Situación de la educación en Chile, año 2000. Análisis de la VIII Encuesta de Caracterización Socioeconómica Nacional (CASEN 2000). Documento n. 4. Santiago: Mideplan, 2001.

WILLIS, P. Aprendiendo a trabajar: cómo los chicos de clase obrera consiguen trabajos de clase obrera. Madrid: Akal, 1988.

\section{Correspondência}

Oscar Dávila León - Centro de Estudios Sociales CIDPA - Condell 1231 Piso 4 Valparaíso Chile. E-mail: oscar@cidpa.cl / felipe@cidpa.cl

Recebido em 17 de novembro de 2010

Aprovado em 26 de janeiro de 2011 